Case Report

\title{
CAVITY IN THE LUNG: A RARE CASE OF BURKHOLDERIA CEPACIA INFECTION
}

\author{
Suresh G. ${ }^{1}$, Rama Prakasha S. ${ }^{2}$, Giridhar B.H. ${ }^{3} \&$ Shama Prakash K. ${ }^{4}$ \\ ${ }^{1}$ Associate Professor, ${ }^{2}$ Assistant Professor, ${ }^{4}$ Assistant Professor, Department of M edicine, ${ }^{3}$ Assistant Professor, \\ Correspondemce: \\ Suresh G., \\ Associate Professor, Department of M edicine, K.S. Hegde M edical Academy, \\ Nitte University, Mangalore - 575 018, Karnataka \\ E-mail : sureshbhatgm@gmail.com
} Department of Respiratory Medicine, K.S. Hegde M edical Academy, Nitte University, M angalore - 575 018, Karnataka

\begin{abstract}
:
An elderly patient was evaluated for fever and cough of three weeks duration. Chest X-ray revealed a thin walled cavity in the right parahilar region. Sputum Acid Fast Bacilli was negative and sputum culture has grown multidrug resistant Burkholderia Cepacia sensitive to carbapenams only. This is a rare case report of community acquired B. Cepacia infection in an individual wherein the clinical presentation was mimicking smear negative pulmonary tuberculosis, but the patient has shown a complete clinical and radiological response to imipenam.
\end{abstract}

Keywords: Berkholderia cepacia, Immunocompetent, Pneumonia

\section{Introduction:}

B. cepacia is a lung pathogen usually in patients with cystic fibrosis. ${ }^{[1,2]}$ It is also described as an important cause of multi-drug resistant nosocomial infection, but infections in immunocompetent patients are extremely rare. Reported cases of B. Cepacia lung infection in immunocompetent individuals include pneumonia and pyopneumothorax. ${ }^{[3-5]}$ Rare cases of acute non-pulmonary B. cepacia infection have also been described in immunocompetent patients. ${ }^{[6]}$ This report is about a patient with no underlying lung disease or systemic illness diagnosed as communityacquired B. cepacia pneumonia mimicking tuberculosis or malignancy, which resolved completely with carbapenem treatment.

\section{Case report :}

A 63 year old male agriculturist was admitted to hospital Access this article online Quick Response Code

\begin{tabular}{|c|}
\hline 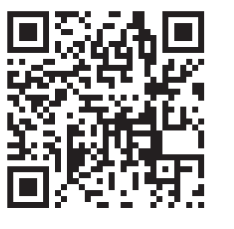 \\
\hline
\end{tabular}
with complaints of high grade intermittent fever and cough with expectoration of three weeks duration. His fever was associated with chills and occurred mostly during evenings, and relieved with sweating. There was history of weight loss of $3 \mathrm{~kg}$ in last three weeks. There was no other significant past medical history. He was non-alcoholic and had history of smoking of 30 pack years. On examination he was emaciated with BMI of $19 / \mathrm{m}^{2}$. Respiratory system examination revealed barrel shaped chest (emphysematous). There was bilateral wheeze with crackles in right infra- clavicular area. Examination of other systems was unremarkable.

Investigations revealed total leucocyte count of 17,600 cells $/ \mathrm{mm}^{3}$, ESR of $55 \mathrm{~mm}$ in one $\mathrm{hr}$. Blood urea was $54 \mathrm{mg} / \mathrm{dl}$ and creatinine was $1.6 \mathrm{mg} / \mathrm{dl}$ on admission, chest $X$-ray showed a cavitatory lesion along hilar area on right

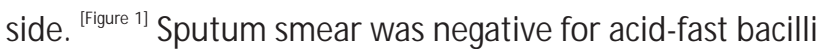
(AFB). He was initially treated with ceftriaxone, azithromycin and amikacin in view of leucocytosis and toxic condition, and there was no clinical response. Sputum culture grew Burkholderia cepacia (heavy growth) and was sensitive only to carbapenemes - meropenam and imepenam. He was treated with imepenam and responded clinically in three to four days with resolution of toxaemia and subsidence of fever. His follow-up chest roentogram after four weeks showed resolution of lung lesion, ${ }^{\left[{ }^{[i g u r e ~ 1]}\right.}$ and 
a complete radiological resonse at the end of six weeks.

\section{Discussion :}

B. Cepacia is a complex group of aerobic gram negative bacilli and infection is commonly described in patients with cystic fibrosis or chronic granulomatous disease with fatal outcome. ${ }^{[1,2]}$ It is very rarely known to cause community acquired infections in immunocompetent individuals. It is also implicated in nosocomial infections, ${ }^{[7]}$ and exhibits high intrinsic antibiotic resistance to most of the available antibiotics. ${ }^{[8,}$ 9] Firstly Waterer et al. described a community-acquired pneumonia caused by $B$. cepacia in healthyadult. ${ }^{[5]}$

The patient in question had fever with productive cough of three weeks duration with a well defined cavity in the right lung. His ESR was elevated and sputum AFB was negative. The sub-acute course of illness with productive cough, evening rise of temperature, sweating, weight loss, elevated ESR and the radiological picture of cavity was pointing towards a diagnosis of smear negative pulmonary tuberculosis. Due to negative sputum smears for AFB, cavitating lung lesion along with his smoking history and advanced age, malignancy was also strongly considered in the differential diagnosis. However, sputum culture grew B. Cepacia and the organism was resistant to all antibiotics
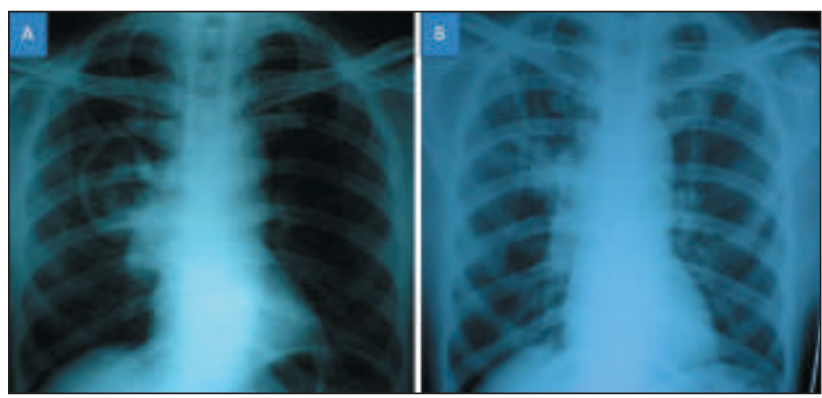

Figure 1: A- Chest $\mathrm{X}$-ray PA view showing cavitatory lesion along the parahilar region on the right side. B- Partial resolution of the lesion on the right side after four weeks of antibiotic therapy.

\section{References:}

1. Lynch JP. Burkholderia cepacia complex: impact on the cystic fibrosis lung lesion. Semin Respir Crit Care M ed 2009;30:596-610.

2. Coutinho HD. Burkholderia cepacia complex: virulence characteristics, importance and relationship with cystic fibrosis. Indian J Med Sci 2007;61:422-9.

3. Pujol, M, Corbell, X, Carratala, J, Gudiol F. Community-acquired bacteremic Pseudomonas cepacia pneumonia in an routinely used, and was susceptible only to carbapenems; to which patient showed a complete clinical and radiological response. Because of its inherent resistance to many antibiotics the organism can be difficult to treat. It has been shown that the organism has a remarkable potential as an agent for both biodegradation and biocontrol, thus it is being considered as a plantgrowthpromoting rhizobacterium. ${ }^{[10]}$ Used as a pesticide and biofertilizer in agriculture, B. cepacia can be transmitted to humans via contaminated agricultural products. Its development in out-of-hospital settings and immunocompetent cases may possibly be attributed to environmental exposure, probably from agricultural produce, like in this case. Otherwise, in non-cystic fibrosis patients B cepacia pneumonia is characteristically a hospital acquired infection in the intubated or immunocompromised patients.

The unusual reporting of such case highlights the presence of rare organism as a cause of lung infection, which was previously considered as merely an opportunistic infection factor, and this is a point of concern. A high index of suspicion and prompt treatment with appropriate antibiotic as per culture and sensitivity is needed to prevent mortality.

immunocompetent host. Clin Infect Dis 1992;15:887-8.

4. Suman SK, Hariharan R, Kiran C, Mukhyaprana P. A rare case of community acquired Burkholderia cepacia infection presenting as pyopneumothorax in an immunocompetent individual. Asian Pacific Journal of Tropical Biomedicine 2012;166-8.

5. Waterer GW, Jones CB, Wunderink RB. Bacteremic communityacquired pneumonia in an immunocompetent adult due to Burkholderia cepacia. Chest 1999;116:1842-3.

6. Hobson R, Gould J, Govan J. Burkholderia (Pseudomonas) cepacia as a cause of brain abscess secondary to chronic supperative otitis externa. EurJ Clin M icrobiol Infect Dis 1995;14:908-11.

7. Bressler AM, Kaye KS, LiPuma JJ, Alexander BD, M oore CM, Reller LB, et al. Risk factors for Burkholderia cepacia complex bacteremia among intensive care unit patients without cystic fibrosis: a case-control study. Infect Control Hosp Epidemiol 2007;28:951-8.

8. Slama TG. Gram-negative antibiotic resistance: there is a price to pay. Crit Care 2008;12 Suppl 4

9. Araque-Calderon $Y, M$ iranda-Contreras $L$, Rodriguez-Lemoine $V$, Palacios-Pru EL. Antibiotic resistance patterns and SDS-PAGE protein profiles of Burkholderia cepacia complex isolates from nosocomial and environmental sources in Venezuela. Med Sci Monit 2008;14:4955.

10. Govan JRW, Hughes JE, Vandamme P. Burkholderia cepacia:medical, taxonomic and ecological issues.J M ed M icrobiol 1996;45:395-407. 